\title{
X-ray diffraction study of the pressure-induced bcc-to-hcp phase transition in the highly magnetostrictive $\mathrm{Fe}_{0.81} \mathrm{Ga}_{0.19}$ alloy
}

\author{
Muhtar Ahart, ${ }^{1}$ Christopher DeVreugd, ${ }^{2}$ Jiefang Li, ${ }^{2}$ Dwight Viehland, ${ }^{2}$ Peter M. Gehring, ${ }^{3}$ and Russell J. Hemley ${ }^{1}$ \\ ${ }^{1}$ Geophysical Laboratory, Carnegie Institution of Washington, Washington, DC 20015, USA \\ ${ }^{2}$ Department of Materials Science and Engineering, Virginia Tech, Blacksburg, Virginia 24061, USA \\ ${ }^{3}$ NIST Center for Neutron Research, National Institute of Standards and Technology, Gaithersburg, Maryland 20899-6100, USA
}

(Received 19 October 2012; revised manuscript received 13 June 2013; published 7 November 2013)

\begin{abstract}
High-pressure x-ray diffraction measurements were performed at room temperature on single crystals of the highly magnetostrictive alloy $\mathrm{Fe}_{0.81} \mathrm{Ga}_{0.19}$ (galfenol). This alloy has a bcc crystal structure at ambient pressure but undergoes a bcc-to-hcp phase transition at $24 \mathrm{GPa}$ on compression. A large hysteresis loop is observed in which the reversed transition occurs at $13 \mathrm{GPa}$ on decompression. The midpoint of this transition is $18.5 \mathrm{GPa}$. The measured bulk modulus of this material is $182( \pm 17) \mathrm{GPa}$, which is comparable to that of pure iron. As with iron, the hcp structure of the alloy can be derived from a compression of the bcc lattice along [001] that is accompanied by shearing along [110]. Our results indicate that the addition of Ga shifts the bcc-to-hcp transition from $13 \mathrm{GPa}$ in pure iron to $18.5 \mathrm{GPa}$, and we speculate that this is due to the larger atomic radius of Ga. A uniaxial loading of $3 \mathrm{GPa}$ completely suppresses the diffuse scattering in $\mathrm{Fe}_{0.81} \mathrm{Ga}_{0.19}$. We ascertain that the magnetostrictive properties of the alloy are reduced under pressure.
\end{abstract}

DOI: 10.1103/PhysRevB.88.184102

PACS number(s): 75.80.+q, 75.50.Bb, 64.70.Nd

\section{INTRODUCTION}

Iron and its alloys are of fundamental interest and have been of great technological importance. As a result, significant efforts have been devoted to studying and optimizing the physical and chemical properties of these materials. ${ }^{1-4}$ The behavior of iron at high pressures and high temperatures is also a key problem in the geophysical sciences ${ }^{5-13}$ because iron is the primary component of the Earth's liquid outer core and solid inner core. Galfenol, a new class of iron-based, magnetic $\mathrm{Fe}_{1-x} \mathrm{Ga}_{x}$ alloy that exhibits giant magnetostriction, has attracted considerable attention because of its potential for use in mechanical devices. ${ }^{14-18} \mathrm{Fe}_{1-x} \mathrm{Ga}_{x}$ alloys with $0.15<$ $x<0.27$ display giant magnetostriction and offer an attractive alternative to conventional piezoelectric ceramics in active mechanical applications, where resistance to fracture is an important criterion. The addition of nonmagnetic Ga into the bcc, $\alpha$-Fe phase is known to produce several crystal structures in the $\mathrm{Fe}_{1-x} \mathrm{Ga}_{x}$ phase diagram, including the chemically disordered bcc (or A2) phase, the ordered bcc $\mathrm{DO}_{3}\left(\mathrm{Fe}_{3} \mathrm{Ga}\right)$ phase, the ordered bcc $\mathrm{B}_{2}$ (FeGa) phase, and the fcc $\mathrm{LI}_{2}$ $\left(\mathrm{Fe}_{3} \mathrm{Ga}\right)$ phase. ${ }^{16}$ Maximum values of the magnetostriction coefficient $\left(\frac{3}{2} \lambda_{100}\right)$ of $400 \mathrm{ppm}$ have been observed in bulk single crystals with $x=0.19 .{ }^{14-17}$ This is more than one order of magnitude higher than that for pure $\alpha$-Fe. It is also interesting to note that $\mathrm{Fe}_{1-x} \mathrm{Ga}_{x}$ alloys display dramatic elastic softening within the same concentration range as the appearance of giant magnetostriction. ${ }^{19}$ There is evidence that this enhancement is not an intrinsic property of a homogeneous ferromagnetic phase; rather the value of $\lambda_{100}$ is known to depend on the heat treatment of the sample, as it varies with the cooling rate after annealing and with the annealing temperature. ${ }^{20-25}$

A structurally heterogeneous model ${ }^{25}$ has been proposed to explain the enhanced magnetostriction and elastic softening for $\mathrm{Fe}_{1-x} \mathrm{Ga}_{x}$ alloys. This model assumes that heat treatment produces a structurally and chemically heterogeneous state consisting of coarsening-resistant, nanometer-scale $\mathrm{DO}_{3}$ precipitates within an A2 matrix. Such heterogeneities have been confirmed experimentally ${ }^{21,26}$ and are believed to result from the following sequence of transformations: (i) bcc, $\mathrm{DO}_{3}$ decomposition, followed by (ii) a diffusionless Bain strain that transforms the $\mathrm{DO}_{3}$ nanoprecipitates into a face-centered tetragonal (fct) phase. In addition, recent neutron diffuse scattering measurements ${ }^{21}$ on $\mathrm{Fe}_{1-x} \mathrm{Ga}_{x}$ alloys $(x=0.10$, $0.15,0.19,0.22$, and 0.25 ) as a function of thermal treatment show that strong diffuse scattering that scales with $\lambda_{100}$ is present within the $\mathrm{A} 2$ and $\mathrm{DO}_{3}$ two-phase region $(0.14<x<$ 0.22 ). For single crystals cooled (postgrowth) at $10^{\circ} \mathrm{C} / \mathrm{min}$ the strongest diffuse scattering is observed for the $x=0.19$ composition; that this diffuse scattering is absent for all compositions cooled at $2{ }^{\circ} \mathrm{C} / \mathrm{min}$ confirms that the enhanced magnetostriction is directly related to the presence of an underlying structural heterogeneity. Furthermore, the diffuse scattering intensity exhibits broad asymmetric peaks near (100) and (300). These findings suggest that the coherent nanoprecipitates that form within the A2 matrix are not cubic but rather of tetragonal (or lower) symmetry. ${ }^{21}$

Pressure can be used to tune the physical properties of materials and introduce new phenomena. For example, pure iron undergoes a bcc-to-hcp structural phase transition near $13 \mathrm{GPa} .{ }^{1-4}$ Motivated by a strong interest in understanding the effects of pressure on these materials, we employed highpressure $\mathrm{x}$-ray diffraction techniques to investigate the phase behavior of the $\mathrm{Fe}_{0.81} \mathrm{Ga}_{0.19}$ alloy at $300 \mathrm{~K}$, which exhibits one of the highest values of $\lambda_{100}$. Our goal was to study how the addition of $\mathrm{Ga}$ affects the pressure-induced bcc-to-hcp phase transition in Fe, how pressure affects the diffuse scattering, and to search for new phases, in order to shed new light on the underlying microscopic mechanism responsible for the giant magnetostriction in the alloy.

\section{EXPERIMENTAL METHODS}

Single-crystal samples of $\mathrm{Fe}_{0.81} \mathrm{Ga}_{0.19}$ were prepared using the Bridgman method as previously described. ${ }^{17}$ These 
samples were cooled, postgrowth, at $10^{\circ} \mathrm{C} / \mathrm{min}$. A polished crystal with dimensions $70 \times 70 \times 20 \mu \mathrm{m}^{3}\left(1 \mu \mathrm{m}=10^{-6} \mathrm{~m}\right)$, the two largest faces of which were oriented along [111], was loaded into a diamond anvil cell (DAC) with $\mathrm{Ne}$ as the pressure medium. Another sample of identical composition and heat treatment, and similar size, but with the two largest faces oriented along [001], was loaded into a separate DAC with Ar as the pressure medium. We also prepared two $300 \mu \mathrm{m}$ thick samples with the above two orientations. These two thicker samples were directly sandwiched between diamond anvils without any pressure medium and were used for the diffuse scattering study. A ruby chip was loaded into each cell for pressure calibration. X-ray diffraction measurements were carried out at beamline 16-BM-D (HPCAT) at the Advanced Photon Source, Argonne National Laboratory. A pre-monochromator combined with a $\mathrm{Si}(311)$ crystal provided a monochromatic $(30.577 \mathrm{keV})$ incident $\mathrm{x}$-ray beam with a wavelength of $0.40548 \AA$. A MAR3450 image plate was used to record oscillation photographs. Standard ruby luminescence techniques were used to determine the pressure with an estimated accuracy of $0.2 \mathrm{GPa}$.

\section{RESULTS AND DISCUSSION}

Figure 1(a) shows a typical $x$-ray diffraction image measured on the $20 \mu \mathrm{m}$ thick [001]-oriented $\mathrm{Fe}_{0.81} \mathrm{Ga}_{0.19}$ single crystal at $3.5 \mathrm{GPa}$. The ringlike features in Fig. 1 demonstrate that, even though the original samples were cut from bulk single crystals, the polishing process has introduced a substantial polycrystalline character to the sample. In addition to the powder diffraction rings from the polycrystalline material, strong spotlike features on the rings are seen that originate from the remaining single crystal. The isolated spots indicated by the red arrows are the result of Bragg diffraction from the diamond anvils. Figures 1(b) and 1(c) show how the (110) Bragg peak evolves as a function of pressure. From this one can see that the (110) Bragg peak splits into a triplet above $24 \mathrm{GPa}$, which marks the bcc-to-hcp phase transition. The peaks from the hexagonal phase persist down to $13 \mathrm{GPa}$ on decompression. Therefore the phase transition appears to be first order insofar as it is accompanied by a large hysteresis loop of about $10 \mathrm{GPa}$ in width, and we identify the midpoint of this hysteresis loop as the transition point, which is $18.5 \mathrm{GPa}$. Figure 2(a) includes data from both the $20 \mu \mathrm{m}$-thick [001]- and [111]-oriented samples and displays the pressure dependence of the $d$ spacing associated with the (110), (200), and (210) Bragg peaks, all of which exhibit nonlinear behavior near the transition point (Note: to avoid confusion, only data measured on compression are shown). Similar behavior is observed for pure iron near the transition pressure. Figure 2(b) shows the pressure dependence of the lattice parameters in both the bcc and hep phases and includes data taken on compression and decompression, and Fig. 2(c) shows the pressure dependence of the unit cell volume for the $\mathrm{Fe}_{0.81} \mathrm{Ga}_{0.19}$ alloy including those calculated from uniaxial stress experiments. For comparison we also show the pressure dependence of the unit cell volume for pure iron in Fig. 2(c). Using the third-order Birch-Murnaghan equation of state (EOS), we obtain $V_{0}=24.4( \pm 0.1) \AA^{3}, K_{0}=182( \pm 17)$ $\mathrm{GPa}$, and $K_{0}^{\prime}=5.6( \pm 1.2)$ for $\mathrm{Fe}_{0.81} \mathrm{Ga}_{0.19}$ in the cubic phase,
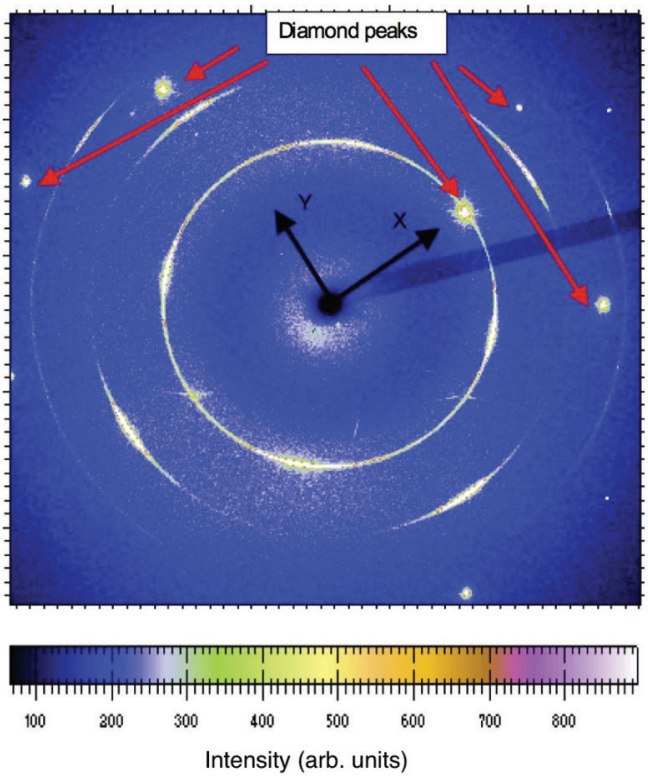

Intensity

(arb. units)

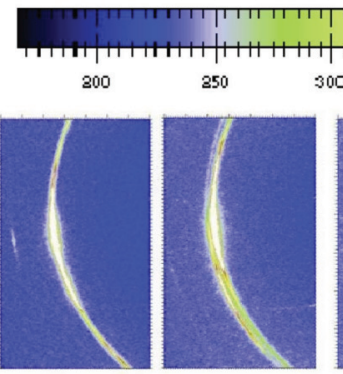

$15 \mathrm{GPa}$

$24 \mathrm{GPa}$

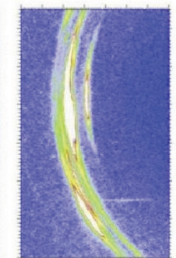

$28 \mathrm{GPa}$

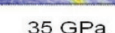

$45 \mathrm{GPa}$

compression

Intensity

(arb. units)

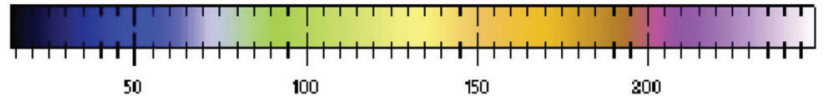

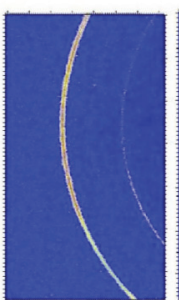

$9 \mathrm{GPa}$

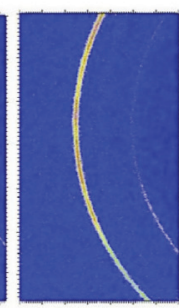

$12.7 \mathrm{GPa}$

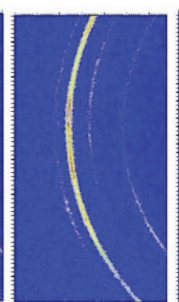

$15 \mathrm{GPa}$

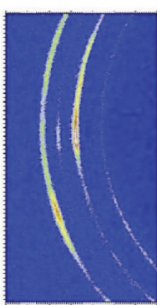

$24 \mathrm{GPa}$

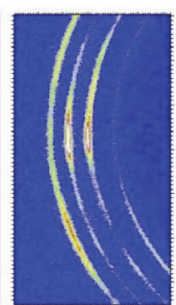

$39 \mathrm{GPa}$
FIG. 1. (Color online) Typical diffraction patterns from the $\mathrm{Fe}_{0.81} \mathrm{Ga}_{0.19}$ alloy. (Top panel) A diffraction pattern at $3.5 \mathrm{GPa}$ ([001]-orientated sample). Strong spots (arrows) are visible from the diamond anvils. The inner ring corresponds to the (110) Bragg peak, the middle ring corresponds to the (210) Bragg peak, and the outer ring corresponds to the (310) Bragg peak. (Middle panel) On compression the (110) Bragg peak from [001]-orientated sample splits into a triplet above $24 \mathrm{GPa}$, which signals the cubic-to-hexagonal phase transition. (Bottom panel) On decompression ([111]-oriented sample), the triplet becomes a single peak below $13 \mathrm{GPa}$, indicating a hexagonal-to-cubic transition. 

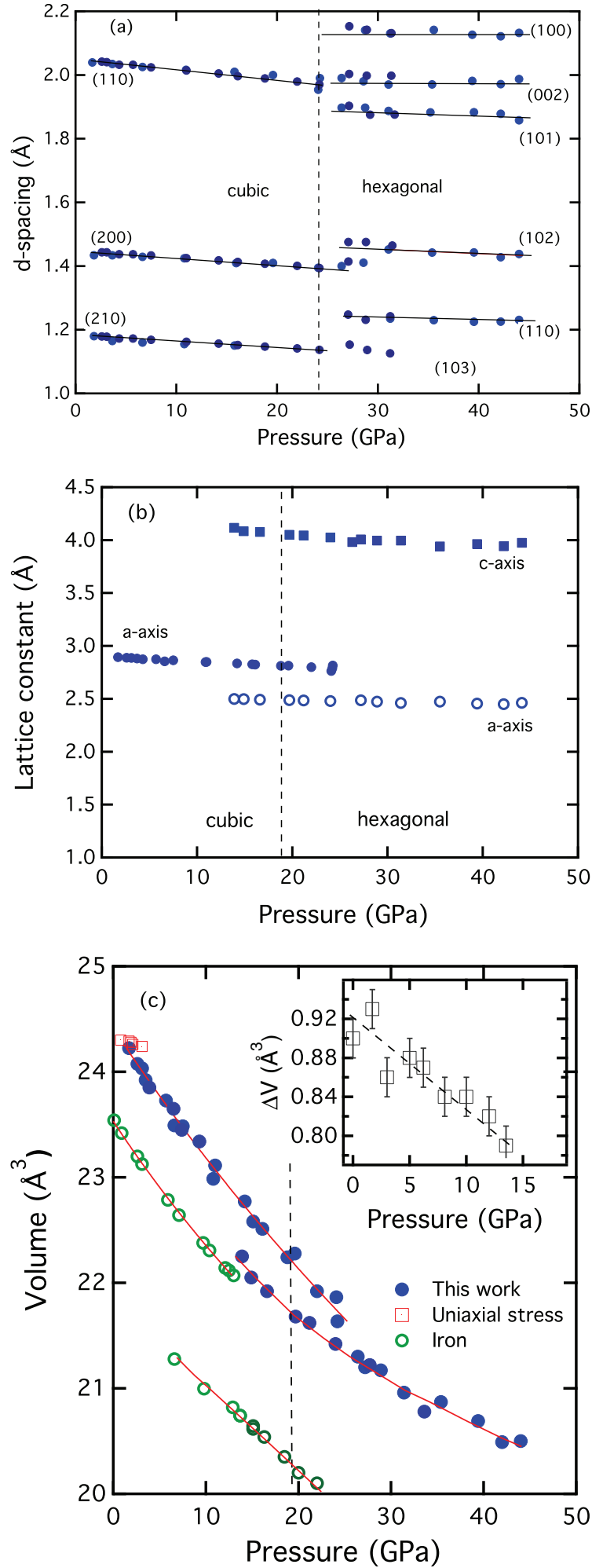

FIG. 2. (Color online) Pressure dependence of the $d$-spacings, lattice constants, and volume for the $\mathrm{Fe}_{0.81} \mathrm{Ga}_{0.19}$ alloy (data shown here are obtained from both samples). (a) $d$-spacings - to avoid the confusion we did not include any data taken on decompression. The size of the error bars is the same as that of the symbols; lines represent a linear fit to the data. For the bcc phase, the fits are $d_{110}=2.0490-0.0032 P, d_{200}=1.4490-0.0022 P$, and $d_{310}=$ 1.1831-0.0019P ; for the hcp phase: $d_{100}=2.1263-0.0001 P, d_{002}=$ $1.9711-0.0001 P, d_{101}=1.8871-0.0013 P, d_{110}=1.4931-0.0013 P$, where $V_{0}$ is the initial volume, $K_{0}$ is the bulk modulus, and $K_{0}^{\prime}$ is the pressure derivative of the bulk modulus at zero pressure. These values are comparable to those determined for pure $\alpha$-Fe, which are $V_{0}=23.5 \AA^{3}$ and $K_{0}=176 \mathrm{GPa}\left[K_{0}^{\prime}\right.$ is fixed at 4 (Ref. 3)].

Our x-ray measurements reveal several interesting facts: (1) The bcc-to-hcp phase transition pressure of $18.5 \mathrm{GPa}$ for single-crystal $\mathrm{Fe}_{0.81} \mathrm{Ga}_{0.19}$ is $6.5 \mathrm{GPa}$ higher than that for pure iron $(13 \mathrm{GPa})$. (2) The pressure of the bcc-to-hcp phase transition on compression and decompression differs by roughly $10 \mathrm{GPa}$ (Fig. 1) whereas for pure iron this difference is only 5 to $6 \mathrm{GPa}$; such hysteretic behavior is characteristic of martensitic transformations. ${ }^{4,5}$ (3) The transition pressures measured using neon and argon as the pressure medium are identical. This indicates that both pressure media maintained a relatively good quasihydrostatic environment. Barge and Boehler ${ }^{6}$ systematically studied pressure medium effects on the bcc-to-hcp transition in pure iron and found that the hysteresis width changes with the medium, particularly under nonhydrostatic conditions. (4) The volume difference between the cubic and hexagonal phases of $\mathrm{Fe}_{0.81} \mathrm{Ga}_{0.19}$ at the transition point is much smaller than that for pure iron; this itself is very interesting and indicates the strong effect of adding Ga. (5) No discernible diffuse scattering was observed from the $20 \mu \mathrm{m}$ thick samples; this is likely due their very small size and to the polishing process, which changed a portion of each single crystal into a polycrystalline (powder) state. However, diffuse scattering was readily apparent (see Fig. 3) in the $300 \mu \mathrm{m}$ thick samples. Since no pressure medium was added to the $300 \mu \mathrm{m}$ thick samples, the compression for these crystals was uniaxial. It should be noted that in our experiments the x-ray beam size was less than $20 \mu \mathrm{m}$, and during nonhydrostatic experiments we collected data only from the center of the DAC. The pressure distribution in this small region is rather homogeneous (hence the pressure can be determined precisely), and the pressure will decrease to almost zero at the edge of the diamond anvil cell. If the center pressure were $3 \mathrm{GPa}$, then the pressure gradient would be $3 \mathrm{GPa} / 150$ $\mu \mathrm{m}$ (the diamond culet is $300 \mu \mathrm{m}$ ) or $0.02 \mathrm{GPa} / \mu \mathrm{m}$. Thus within the X-ray beam size of $20 \mu \mathrm{m}$ we expect no more than a $0.4 \mathrm{GPa}$ pressure difference.

There are several possible origins of this diffuse scattering that are relevant to $\mathrm{FeGa}$ alloys. One is thermal diffuse scattering, which results from the low-energy phonons of

and $d_{111}=1.2341-0.0002 P$. (b) Pressure dependence of the lattice parameters of the cubic and hexagonal cells. Solid circles represent cubic lattice parameters, open circles and solid squares represent hexagonal lattice parameters a and c, respectively. (c) EOS. Solid circles represent data from this work and solid curves are fits to a third-order Birch-Murnaghan equation of state. Open circles are volume data for pure iron adapted from Refs. 3, 6, 11. Open squares are volume data obtained from uniaxial compression up to $3.3 \mathrm{GPa}$. Both datasets collected on compression and decompression are shown in (b) and (c). The inset shows the pressure dependence of the volume difference between $\mathrm{Fe}_{0.81} \mathrm{Ga}_{0.19}$ and pure iron. It should be noted that the volume difference between the cubic and hexagonal cells of the $\mathrm{Fe}_{0.81} \mathrm{Ga}_{0.19}$ sample is smaller than that of pure iron. 

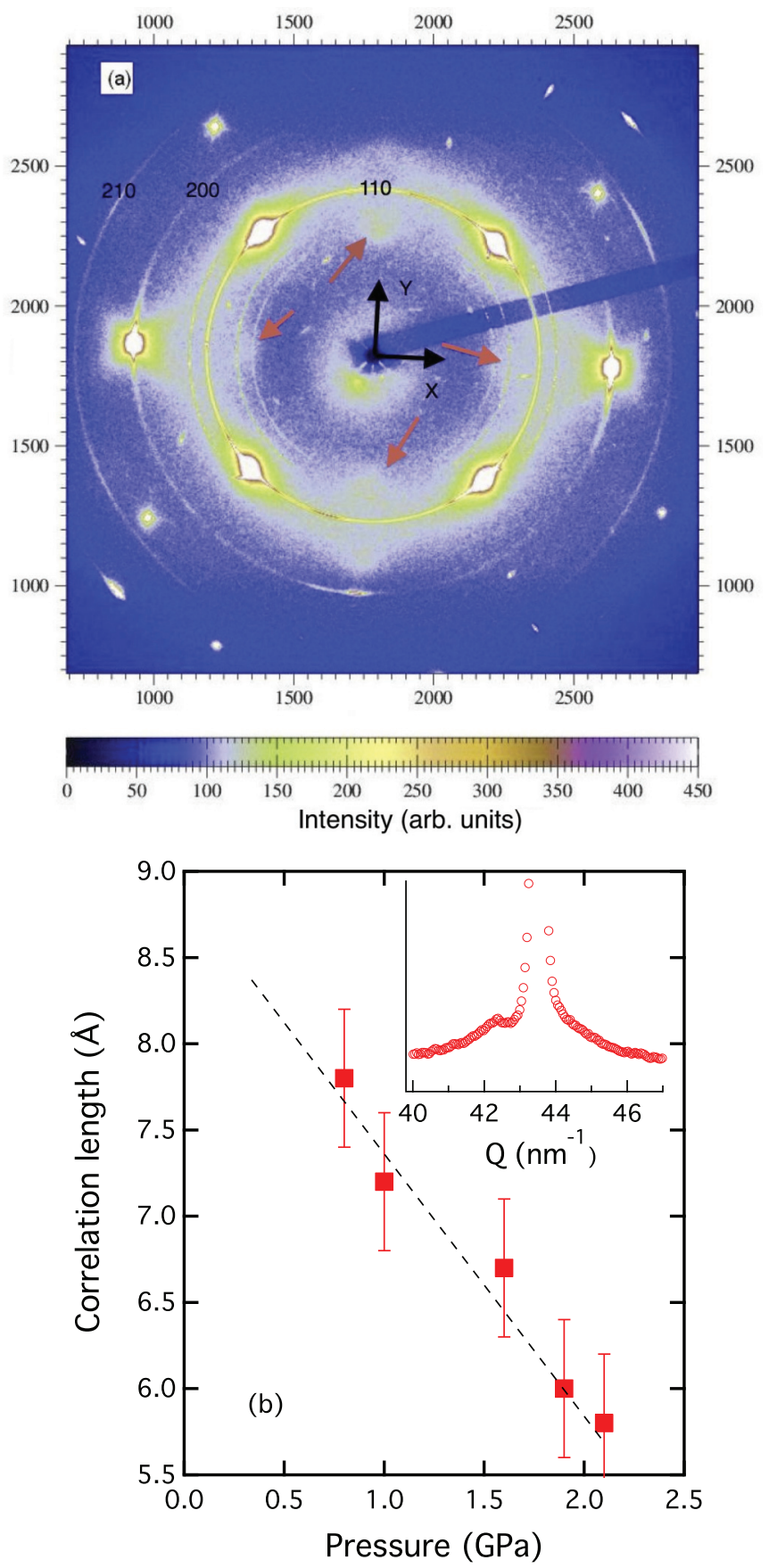

FIG. 3. (Color online) (a) Diffuse scattering from [001]-oriented and $300 \mu \mathrm{m}$ thick $\mathrm{Fe}_{0.81} \mathrm{Ga}_{0.19}$ alloy at $0.8 \mathrm{GPa}$ stress. No pressure medium is present. Diffuse superstructure peaks are visible at the four equivalent (100) positions and are indicated by arrows (one is located directly under the "110" label). (b) Pressure dependence of the radial correlation length calculated by deconvoluting the integrated peak from the diffuse scattering near the (200) Bragg peak in (a). The error bars are estimated from the scatter of the data.

the crystal lattice. A second is substitutional disorder. We exclude these two origins because they should not exhibit much pressure dependence whereas the diffuse scattering we observe in Fig. 3(a) vanishes at $3 \mathrm{GPa}$. A third possible origin is $\mathrm{DO}_{3}$ nanoprecipitates. As shown in Fig. 1(a) of Ref. 21, the diffuse scattering associated with these nanoprecipitates decorates the (100) and related reciprocal lattice vectors. It is also rather featureless, being essentially isotropic in reciprocal space. It is also present near (200) as we show in Fig. 3(a). Because we also see very faint, broad diffuse peaks (shown in green) at all four (100) reciprocal lattice positions in Fig. 3(a), we conclude that this diffuse scattering is associated with the $\mathrm{DO}_{3}$ precipitates. That this diffuse scattering disappears above $3 \mathrm{GPa}$ indicates that uniaxial pressure is able to suppress the nanometer-scale precipitates. Cao et al. ${ }^{21}$ showed that this diffuse scattering intensity correlates with the strength of the magnetostriction coefficient, and that $\mathrm{Fe}_{1-x} \mathrm{Ga}_{x}$ crystals cooled postgrowth at $10^{\circ} \mathrm{C} / \mathrm{min}$ exhibit the strongest diffuse scattering and the largest magnetostriction effect at $x=0.19$. Although the precise origin of diffuse scattering in $\mathrm{Fe}_{1-x} \mathrm{Ga}_{x}$ alloys is still under debate, we believe that the diffuse scattering arises from short-range correlations between the local structures that influence the magnetostriction effect. ${ }^{21}$ Our results qualitatively agree with those of Atulasimha et al., ${ }^{24}$ who studied the effect of uniaxial stress on magnetostriction for $\mathrm{Fe}_{1-x} \mathrm{Ga}_{x}$ alloys $(0.15 \leqslant x \leqslant 0.21)$. They found that uniaxial stress reduces the magnetostriction, but they only made measurements up to $80 \mathrm{MPa}(0.08 \mathrm{GPa})$. In this low-pressure regime, despite the suppression, the alloys still showed substantial magnetostriction. In our study, we applied over 500 times more stress and found that the diffuse scattering disappears around $3 \mathrm{GPa}$. The concurrent decrease of the magnetostriction and the diffuse scattering through the application of uniaxial stress provides yet another experimental link between these two properties. Our results clearly indicate that uniaxial pressure suppresses the diffuse scattering, which indicates a decrease in the magnetostriction in the Fe-Ga alloy. Thus one may thus consider using uniaxial stress at very modest pressures to control the magnetostriction.

Martensitic transformations are termed "diffusionless" because they do not involve the movement of atoms over distances larger than the lattice dimensions. ${ }^{1-4}$ This type of phase transformation is also termed "displacive." structure can be derived from the bcc structure through a relatively minor distortion. ${ }^{3,5}$ In the case of $\mathrm{Fe}_{1-\mathrm{x}} \mathrm{Ga}_{\mathrm{x}}$ alloys, it is not known exactly how $\mathrm{Ga}$ replaces $\mathrm{Fe}$ in the bcc structure. However, we assume that $\mathrm{Ga}$ replaces one of the center $\mathrm{Fe}$ atoms as shown in Fig. 4 (e.g., Ref. 5). Thus, by analogy with the phase transition in pure iron, we can qualitatively explain the pressure-induced bcc-to-hcp phase transition in $\mathrm{Fe}_{0.81} \mathrm{Ga}_{0.19}$. The hcp structure [Fig. 4(b)] of $\mathrm{Fe}_{1-x} \mathrm{Ga}_{x}$ alloys also can be derived from the bcc structure by (i) compressing the bcc [Fig. 4(a)] unit cell along [001], thus reducing the $d$ spacing of the bcc (002) plane to form the hcp (120) plane and expanding the $d$ spacing of the bcc (110) plane to form the hcp (100) plane; (ii) translating alternate bcc (110) layers in the [110] direction, producing the hcp (101) plane; and (iii) preserving the two-layer (A and $\mathrm{B}$ ) repeat distance in the bcc structure; that is, keeping the $d$ spacing of the bcc (110) plane equal to that of the hcp (002) plane. However, we still cannot rule out the effects of diffusion of $\mathrm{Ga}$ atoms on the phase transition based only on our experimental measurements. There could be some diffusion even at room temperature, and strains could develop from strength effects. (Due to the addition of $\mathrm{Ga}$ into the Fe lattice, the tensile or compressive strength of iron as well as the local structures will 


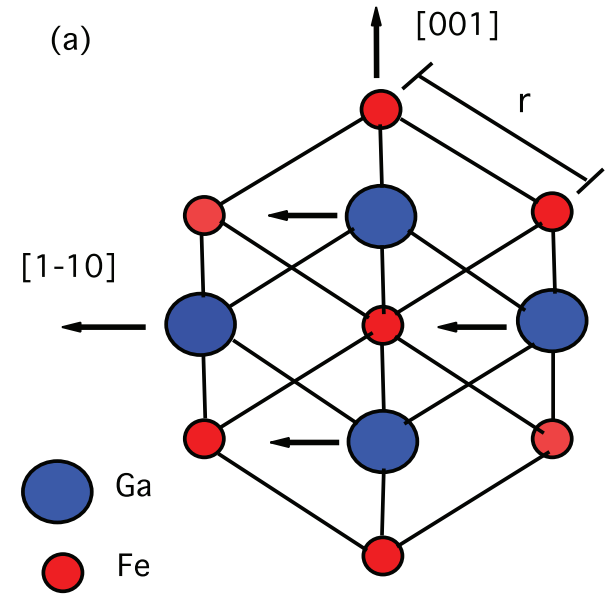

(b)

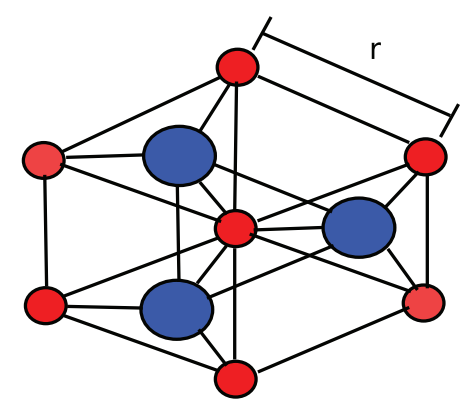

FIG. 4. (Color online) Schematic diagram indicating a possible mechanism for the bcc-hcp phase transition in $\mathrm{Fe}_{0.81} \mathrm{Ga}_{0.19}$. (a) [110] projection of the bcc phase. (b) [001] projection of the hcp phase. Only two layers (red: Fe, blue: Ga) are shown in the diagram. The bcc phase is transformed from the hcp phase via compression along [001] and shearing of the layer along [110].

change accordingly, and these conditions would induce strain.) Nevertheless, it should be emphasized that the transition is accompanied by a large hysteresis loop, which is characteristic of a martensitic transformation.

Although the mechanism underlying the bcc-to-hcp transition is likely similar for both pure iron and the Fe-Ga alloy, the transition pressure is $18.5 \mathrm{GPa}$ for the alloy at room temperature, which is nearly twice that $(13 \mathrm{GPa})$ for pure Fe. The reason for this large difference is likely due to the fact that the atomic radius of $\mathrm{Ga}(135 \mathrm{pm})$ is larger than that for $\mathrm{Fe}(125 \mathrm{pm}){ }^{28}$ Although the atomic radius is a loosely defined concept, in metallic states, Ga adopts an orthorhombic structure under ambient conditions in which each atom has one nearest neighbor and six next-nearest neighbors. This could be why Ga has larger atomic radius; on the other hand, Fe has a bcc structure in which each Fe is eightfold coordinated; in addition, as shown in Fig. 2.1 of Ref. 27, the molar volume of $\mathrm{Ga}$ is also larger than that of $\mathrm{Fe}$ at ambient conditions. ${ }^{28}$ The addition of $\mathrm{Ga}$ into the iron lattice will cause some structural change at least locally. Such a change should induce an excess volume as suggested in Ref. 25; this excess volume is evident from the comparison between the initial volumes of $\mathrm{Fe}$ and $\mathrm{Fe}_{0.81} \mathrm{Ga}_{0.19}$ shown in the inset to Fig. 2(c); we speculate that this extra volume may correspond to the averaged volume of $\mathrm{DO}_{3}$ nanoprecipitates. During compression along the [001] direction of the bcc lattice, a higher pressure is required for the alloy to reach the "shearing" stage of the bcc-to-hcp transformation mechanism due to the larger atomic radius of $\mathrm{Ga}$. The pressure-induced phase transition will therefore be delayed to a higher pressure in the $\mathrm{Fe}-\mathrm{Ga}$ alloy than that in pure iron.

Of course the replacement of Fe with $\mathrm{Ga}$ is most likely far more complex than that suggested by Fig. 4. Neutron scattering results on $\mathrm{Fe}_{0.81} \mathrm{Ga}_{0.19}$ are consistent with a structurally ${ }^{21}$ and magnetically ${ }^{26}$ heterogeneous state consisting of a dispersion of nanometer-sized precipitates embedded within an A2 matrix phase. The existence of such a heterogeneous state is consistent with the theoretical model of Khachaturyan and Viehland, ${ }^{25}$ according to which a coarsening-resistant dispersion of $\mathrm{DO}_{3}$ nanoparticles is formed within the composition range adjacent to the A2 phase solubility limit. Even though the coexisting A2 and $\mathrm{DO}_{3}$ phases are both cubic, the volume of the Ga-enriched $\mathrm{DO}_{3}$ precipitates $\left(\mathrm{Fe}_{3} \mathrm{Ga}\right)$ is larger than that of the $\mathrm{A} 2$ matrix from which it formed. This is a direct consequence of the fact that the atomic radius of $\mathrm{Ga}$ is greater than that of Fe. The Ga-enrichment-induced extra volume produced by the crystal lattice expansion generates elastic strain, which suppresses the $\mathrm{DO}_{3}$-phase precipitation in the coherent state. The size $D$ of the $\mathrm{DO}_{3}$ precipitates can then be estimated from the fact that $D$ should be close to (but less than) the amount $a_{A 2} / 2$ that has to be removed by an excess vacancy to reduce the transformationhindering stress. This leads to the following inequality:

$$
D \leqslant \frac{a_{A 2}^{2}}{2\left(\frac{a_{D O 3}}{2}-a_{A 2}\right)} .
$$

According to this inequality, the estimated size of the $\mathrm{DO}_{3}$ precipitates is about $10 \mathrm{~nm}$ for the $\mathrm{Fe}_{0.81} \mathrm{Ga}_{0.19}$ alloy under ambient conditions. On the other hand, pressure can tune the structural properties of Fe-Ga alloys. Figure 3(a) shows the presence of strong diffuse scattering that exhibits a structure factor consistent with that expected for the $\mathrm{DO}_{3}$ structure. Thus, we can qualitatively discuss how pressure affects the correlations between the nanometer-sized clusters based on our observations of the pressure dependence of diffuse scattering. Similar examples in which pressure suppresses the diffuse scattering have been reported in several relaxor ferroelectric materials. ${ }^{29,30}$ In fact, not only is the pressure dependence of the diffuse scattering similar between relaxors and galfenol, but so are the respective phase diagrams. For example, the phase diagram of $\mathrm{Pb}\left(\mathrm{Mg}_{1 / 3} \mathrm{Nb}_{2 / 3}\right) \mathrm{O}_{3}-x \mathrm{PbTiO}_{3}$ solid solutions ${ }^{31}$ consists of a rhombohedral $R 3 m$ phase for $x<0.32$, an intermediate monoclinic $C m$ phase for $0.32<$ $x<0.37$, and a tetragonal $P 4 m m$ phase for $x>0.37$; on the other hand, the phase diagram of $\mathrm{Fe}_{1-x} \mathrm{Ga}_{x}$ alloys consists of the bcc phase for $x<0.15$, an intermediate phase for $0.15<$ $x<0.25$, and a tetragonal phase for $x>0.25$. The details of the phase diagram are more complex. We therefore applied a method similar to that described in Ref. 28, and used a deconvolution method to obtain the wave vector linewidth (Lorentzian function) of the diffuse scattering from which we calculated the radial correlation length. Figure 3(b) shows the pressure dependence of this correlation length under uniaxial conditions, and the inset shows a linear cut through the (200) Bragg peak taken approximately along [100]. It is obvious that the correlation length of the diffuse scattering decreases with 
pressure. It should be noted that the correlation length for our sample is about $8 \AA$ at $0.8 \mathrm{GPa}$; this is ten times smaller than the $\mathrm{DO}_{3}$ size estimated from inequality (1) at ambient conditions. One possible reason for this is that the correlations have already been substantially reduced at this pressure. In fact, this result is consistent with the observation of Ref. 3, which shows that increasing uniaxial stress first enhances the magnetostriction effect and then suppresses it. The strain rate drops to almost half of its original (zero-pressure) value at $0.08 \mathrm{GPa}^{24}$

\section{CONCLUSIONS}

We have performed high-pressure $\mathrm{x}$-ray diffraction studies of $\mathrm{Fe}_{0.81} \mathrm{Ga}_{0.19}$ single crystals at room temperature up to $45 \mathrm{GPa}$. Our results show that $\mathrm{Fe}_{0.81} \mathrm{Ga}_{0.19}$ undergoes a bcc-to-hcp phase transition at $18.5 \mathrm{GPa}$ and displays a large hysteresis loop in which the decompression transition occurs at $13 \mathrm{GPa}$; this is a signature of a martensitic transformation. We proposed a possible mechanism for the transition in which the hcp structure can be derived from the bcc lattice through a compression along the [001] direction of the bcc lattice, followed by shear along the [110] direction. The mechanism is similar to that of pure iron. We speculate that the larger atomic size of $\mathrm{Ga}$ is responsible for the higher bcc-to-hcp transition pressure of $18.5 \mathrm{GPa}$ compared to that $(13 \mathrm{GPa})$ for pure iron. The bulk modulus of the $\mathrm{Fe}_{0.81} \mathrm{Ga}_{0.19}$ alloy is $K_{0}=$ $182( \pm 17) \mathrm{GPa}$, and its pressure derivative is $K_{0}^{\prime}=5.6( \pm 1.2)$. These numbers are comparable with those of pure iron. In addition, we studied the pressure dependence of the diffuse scattering in $\mathrm{Fe}_{0.81} \mathrm{Ga}_{0.19}$ under uniaxial pressure. We find that a modest uniaxial stress of $\sim 3 \mathrm{GPa}$ is sufficient to suppress the diffuse scattering, which originates from the presence of $\mathrm{DO}_{3}$ nanoprecipitates. Consequently the $\mathrm{DO}_{3}$ nanoprecipitates play no role in the bcc-to-hcp phase transition.

\section{ACKNOWLEDGMENTS}

This work was sponsored by the Carnegie/DOE Alliance Center (CDAC, DE-FC03-03NA00144). Support from the Office of Naval Research through Grant No. N00014-1010292 is also acknowledged. Use of the Advanced Photon Source was supported by the US Department of Energy under Contract No. DE-AC02-06CH11357. HPCAT operations are supported by CIW, CDAC, UNLV, and LLNL through funding from DOE-NNSA and DOE-BES, with partial instrumentation funding by NSF.
${ }^{1}$ D. Bancroft, E. L. Peterson, and S. Minshall, J. Appl. Phys. 27, 291 (1956).

${ }^{2}$ H. Jones, Mater. Sci. Eng. 5, 1 (1969).

${ }^{3}$ H. K. Mao, W. A. Bassett, and T. Takahashi, J. Appl. Phys. 38, 272 (1967).

${ }^{4}$ P. M. Giles, M. H. Longenbach, and A. R. Marder, J. Appl. Phys. 42, 4290 (1971)

${ }^{5}$ W. A. Bassett and E. Huang, Science 238, 780 (1987).

${ }^{6}$ H. K. Mao, Y. Wu, L. Chen, J. F. Shu, and A. P. Jephcoat, J. Geophys. Res. 95, 21737 (1990).

${ }^{7}$ N. Von Barge and R. Boehler, High Press. Res. 6, 133 (1990).

${ }^{8}$ R. Boehler, Nature (London) 363, 534 (1993).

${ }^{9}$ H. K. Mao, J. Xu, V. V. Struzhkin, J. Shu, R. J. Hemley, W. Sturhahn, M. Y. Hu, E. E. Alp, L. Vocadlo, D. Alfè, G. D. Price, M. J. Gillan, M. Schwoerer-Böhning, D. Häusermann, P. Eng, G. Shen, H. Giefers, R. Lübbers, and G. Wortman, Science 292, 914 (2001).

${ }^{10}$ C. T. Seagle, A. J. Campbell, D. L. Heinz, G. Shen, and V. B. Prakapenka, J. Geophys. Res. 111, B06209 (2006).

${ }^{11}$ A. Dewaele, P. Loubeyre, F. Occelli, M. Mezouar, P. I. Dorogokupets, and M. Torrent, Phys. Rev. Lett. 97, 215504 (2006).

${ }^{12}$ W. L. Mao, V. V. Struzhkin, A. Q. R. Baron, S. Tsutuui, C. E. Tommaseo, H.-R. Wenk, M. Y. Hu, P. Chow, W. Sturhahn, J. Shu, R. J. Hemley, D. L. Heinz, and H.-K. Mao, J. Geophys. Res. 113, B09213 (2008).

${ }^{13}$ X. Sha and R. E. Cohen, Phys. Rev. B 81, 094105 (2010).

${ }^{14}$ A. E. Clark, J. B. Restorff, M. Wun-Fogle, T. A. Lograsso, and D. L. Schlagel, IEEE Trans. Magn. 36, 3238 (2000).

${ }^{15}$ A. E. Clark, M. Wun-Fogle, J. B. Restorff, and T. Lograsso, Proceedings of the Fourth Pacific Rim International Conference on Advanced Materials and Processing (PRICM4), edited by S. Hanada, Z. Zhong, S. Nam, and R. Wright (Japan Institute of Metals, 2001), p. 1711.
${ }^{16}$ O. Ikeda, R. Kainuma, I. Ohnuma, K. Fukamichi, and K. Ishida, J. Alloys Compd. 347, 198 (2002).

${ }^{17}$ D. Viehland, J. F. Li, T. A. Lograsso, A. Ross, and M. Wuttig, Appl. Phys. Lett. 81, 3185 (2002).

${ }^{18}$ S. Ostanin, J. B. Staunton, S. S. A. Razee, C. Demangeat, B. Ginatempo, and Ezio Bruno, Phys. Rev. B 69, 064425 (2004).

${ }^{19}$ J. L. Zarestky, V. O. Garlea, T. A. Lograsso, D. L. Schlagel, and C. Stassis, Phys. Rev. B 72, 180408 (2005).

${ }^{20} \mathrm{~S}$. Bhattacharyya, J. R. Jinschek, A. Khachaturyan, H. Cao, J. F. Li, and D. Viehland, Phys. Rev. B 77, 104107 (2008).

${ }^{21}$ H. Cao, P. M. Gehring, C. P. Devreugd, J. A. Rodriguez-Rivera, J. Li, and D. Viehland, Phys. Rev. Lett. 102, 127201 (2009).

${ }^{22}$ J. L. Zarestky, O. Moze, J. W. Lynn, Y. Chen, T. A. Lograsso, and D. L. Schlagel, Phys. Rev. B 75, 052406 (2007).

${ }^{23}$ H. Cao, F. Bai, J. Li, D. D. Viehland, T. A. Lograsso, and P. M. Gehring, J. Alloys Compd. 465, 244 (2008).

${ }^{24}$ J. Atulasimha, A. B. Flatau, and J. R. Cullen, Smart Mater. Struct. 17, 025027 (2008).

${ }^{25}$ A. G. Khachaturyan and D. Viehland, Metall. Mater. Trans. A 38, 2308 (2007).

${ }^{26}$ M. Laver, C. Mudivarthi, J. R. Cullen, A. B. Flatau, W.-C. Chen, S. M. Watson, and M. Wuttig, Phys. Rev. Lett. 105, 027202 (2010).

${ }^{27}$ Diffuse X-Ray Reflections from Crystals, edited by W. A. Wooster (Clarendon Press, Oxford, 1962); Diffuse Scattering and the Fundamental Properties of Materials, edited by R. Barabash, G. E. Lee, and P. E. A. Turchi (Momentum Press, 2009).

${ }^{28}$ N. N. Greenwood and A. Earnshaw, Chemistry of the Elements (Pergamon Press, 1984), Chaps. 7 and 25. It should be noted that atomic radius is a loosely defined concept (e.g., Van der Waals, ionic, covalent, and metallic radii). Here we insist on the value of 
the metallic radius of $\mathrm{Ga}(135 \mathrm{pm})$ and $\mathrm{Fe}(126 \mathrm{pm})$ from the above book. For covalent elemental radii see J. C. Slater, J. Chem. Phys. 41, 3199 (1964).

${ }^{29}$ B. Chaabane, J. Kreisel, B. Dkhil, P. Bouvier, and M. Mezouar, Phys. Rev. Lett. 90, 257601 (2003).
${ }^{30}$ M. Ahart, R. E. Cohen, V. Struzhkin, E. Gregoryanz, D. Rytz, S. A. Prosandeev, H.-K. Mao, and R. J. Hemley, Phys. Rev. B 71, 144102 (2005).

${ }^{31}$ B. Noheda, D. E. Cox, G. Shirane, J. Gao, and Z.-G. Ye, Phys. Rev. B 66, 054104 (2002). 